CORRECTION

https://doi.org/10.1038/s41586-018-0142-y

\title{
Author Correction: A series of energetic metal pentazolate hydrates
}

Yuangang Xu, Qian Wang, Cheng Shen, Qiuhan Lin, Pengcheng Wang \& Ming Lu

Correction to: Nature https://doi.org/10.1038/nature23662,

published online 28 August 2017.

In this Letter, under Methods section ' $\left[\mathrm{Na}\left(\mathrm{H}_{2} \mathrm{O}\right)\left(\mathrm{N}_{5}\right)\right] \cdot 2 \mathrm{H}_{2} \mathrm{O}(2)$ ', the description "the intermediate product arylpentazole $(5.000 \mathrm{~g}, 26.18$ mmol)" should have read "the intermediate product sodium salt of arylpentazole $(5.000 \mathrm{~g}, 21.64 \mathrm{mmol})$ ". In the legend of Fig. 3, we add that "All temperature points in the stability study were onset temperatures." to avoid misunderstanding. These corrections have been made online. 